Cahiers de recherches médiévales

\title{
La figure du roi et le point de vue du poète
}

Fonctions de César dans Les Discours de Jules Cesar avant le passage du Rubicon, d'Étienne Jodelle

\section{Emmanuel Buron}

\section{(2) OpenEdition}

\section{Journals}

Édition électronique

URL : https://journals.openedition.org/crm/852

DOI : $10.4000 / \mathrm{crm} .852$

ISSN : 1955-2424

Éditeur

Honoré Champion

Édition imprimée

Date de publication : 30 mars 2006

Pagination : 107-124

ISSN : 1272-9752

Référence électronique

Emmanuel Buron, «La figure du roi et le point de vue du poète », Cahiers de recherches médiévales [En ligne], 13 spécial | 2006, mis en ligne le 03 avril 2009, consulté le 15 décembre 2022. URL : http:// journals.openedition.org/crm/852; DOI : https://doi.org/10.4000/crm.852 


\section{rM}

\section{La figure du roi et le point de vue du poète : fonctions de César dans Les Discours de Jules Cesar avant le passage du Rubicon, d'Étienne Jodelle}

Parler des Discours de Jules Cesar avant le passage du Rubicon, d'Étienne Jodelle, dans le cadre d'un colloque sur César au Moyen-âge et à la Renaissance est à la fois évident et paradoxal' ${ }^{1}$ Évident, parce que le titre de ce poème indique d'emblée qu'il prend pour objet principal un épisode-clé de l'histoire du général romain. En outre, ce texte constitue la plus ambitieuse des entreprises poétiques du poète: selon Charles de la Mothe, qui préface les Euvres et meslanges poetiques, édition des œuvres de Jodelle parue en 1574, un an après la mort de l'auteur, ce poème devait «monter à dix mille vers pour le moins »'. Toutefois, et c'est pourquoi il devient paradoxal d'en parler ici, ce poème, tel qu'il a été publié en 1574, est inachevé ; et si le fragment conservé compte tout de même 2336 vers, il y est fugitivement question de César et du Rubicon au début, avant que, de digression en digression, Jodelle perde ce sujet de vue. Enea Balmas, l'éditeur moderne des œuvres de Jodelle, note donc :

Dans l'état dans lequel il nous est parvenu, il est malaisé d'en comprendre même le titre, car lorsqu'on est arrivé à la fin du fragment que nous possédons, les « discours » du célèbre capitaine romain n'ont pas encore commencé. On ne peut donc se faire aucune idée de ce que Jodelle avait mis dans son poème pour en justifier le titre ${ }^{3}$.

Un rapide résumé du poème permettra d'apprécier tout à la fois la grande dispersion apparente des sujets abordés, et - caractère frappant des Discours comme de toute la poésie jodellienne - l'extrême densité du raisonnement, qui donne au texte la complexité d'un traité poétique. Le poème s'ouvre sur une longue analyse des rapports que le «grand cœur» entretient avec les cours : ce magnanime fuit bien sûr «le service et la suite» des tyrans, mais il fuit aussi les rois, impatient devant la servilité qui règne dans leur entourage, et trop épris de liberté pour supporter la contrainte que son service lui imposerait, d'autant plus grande qu'il servirait volontairement (v. 1-90). Comme néanmoins, il se sent un devoir de servir, il accomplit alors cette mission dans la solitude, par des traités où il veut éclairer le roi (v. 91162). Tel est le cas de Jodelle, qui, après avoir longtemps servi le roi en secret lui adresse aujourd'hui les Discours, en prémisse d'autres œuvres (v. 163-218). Les

\footnotetext{
${ }^{1}$ Les Discours de Jules Cesar avant le passage du Rubicon ont été publiés dans : É. Jodelle, CEuvres et meslanges poetiques, Paris, N. Chesneau et M. Patisson, 1574, fol. $135^{\mathrm{v}}-174^{\mathrm{v}}$; et repris dans É. Jodelle, CEuvres complètes, t. II, éd. E. Balmas, Paris, Gallimard, 1968, p. 293351. Nous donnerons nos références par rapport à ces deux éditions, respectivement désignées en abrégé par $O M P$ et $O . C$.

${ }^{2} O M P$, sig. e ; O.C. I, p. 72.

${ }^{3}$ O.C. II, p. 477. Balmas analyse la matière «mêlée » du poème ibid, p. 479-480.
}

Cahiers de Recherches Médiévales, 13spé, 2006 
Discours porteront d'abord sur César au bord du Rubicon, avant que Jodelle applique cet exemple au roi (v.219-230). Suit une évocation du général à la veille d'entrer en guerre civile (v. 231-250), une analyse de l'hostilité que son approche provoque chez les hommes politiques romains (v. 251-286), puis de la liberté romaine agonisante (v. 287-342). Jodelle s'élève alors jusqu'à la méditation du destin des états, et dégage la loi de vicissitude, qui condamne toute grandeur à la chute (v. 386-440). Il observe cette loi de vicissitude dans la danse des astres autant que dans les états, mais il constate qu'au ciel, il y a toujours un astre ou plusieurs qui exercent leur ascendant, et que jamais tous ne dominent (v. 441-489). Il applique alors cette observation astronomique aux constitutions politiques, et justifie les régimes monarchique et aristocratique contre les régimes démocratiques, au nom d'un principe de grandeur, qui suppose une hiérarchie sociale (v. 490-888). Suit une invective contre les officiers iniques, qui manquent à la justice et accablent le peuple d'impôts (v. 889-1002) ; ce qui incite le poète à se faire satirique, se donnant pour mission de fustiger les vices, afin d'inciter le roi à la poursuite de la grandeur (v. 1003-1052).

Ce point amorce ce qu'on peut considérer comme une deuxième partie dans le texte, que nous résumerons moins précisément. En incitant les rois à chercher la grandeur, Jodelle est amené à souligner l'injustice de la mémoire, qui accorde plus de poids aux erreurs et aux fautes qu'aux belles actions, surtout quand ce vice entraîne la ruine du prince concerné, si bien qu'un vertueux peut être oublié ou méprisé pour une seule erreur ${ }^{4}$. Jodelle illustre ce point par l'analyse des exemples de Sardanapale (v. 1153-1194) et de rois tragiques, puis il s'arrête sur Priam, ce qui le conduit à examiner ce qu'on dit de la guerre de Troie (à partir du v. 1239) et en particulier du combat d'Achille et d'Hector (à partir du v. 1654), à propos duquel il montre la partialité d'Homère qui a été injuste envers Hector, pour mieux exalter Achille, par solidarité nationale (le poète et le héros étaient grecs) et en fonction de la loi de la postérité, qui élève les vainqueurs et accable les vaincus 5 .

Arrivé au terme du poème, le lecteur a d'abord du mal à en embrasser la totalité, mais la cohérence du texte est plus forte qu'il peut d'abord sembler. En effet, Jodelle développe bel et bien le contenu, le plan et la méthode qu'il annonce dans son titre et au début des Discours ; mais il les développe d'une manière surprenante, dont l'inachèvement du texte empêche de mesurer la rigueur. Notre premier travail consistera donc à éclaircir le projet générique des Discours, en élucidant le titre et la démarche qu'il indique : César apparaît alors comme la figure qui donne son unité

\footnotetext{
${ }^{4}$ Evoquant la ruine des Princes, Jodelle note : «c'est un obscur tombeau,/ Si tombeau mesme ils ont, qui pour la fin receuë,/ Peut estre, couvrira la grace qu'ils ont euë / Pour un temps, la faveur des spectateurs, l'honneur,/ Magnificence, pompe, accortesse, et bon heur»; mais ce même tombeau «ne couvrira pas,/ Soit pour la vie, ou bien pour l'horrible trespas,/ Les deffauts d'heur, de sens, de bon cœur, de paroles / Dignes, et dignes faits, advis, les rages, les folles/ Ardeurs, l'horreur honteuse en l'air il vomira,/ Puis par tout l'univers l'air l'éparpillera,/ Tant que le bruit ailé qui fera d'âge en âge / Courir ce qui est pire, en portant grand dommage / A tout bien qu'ils ont eu, portera grand renfort / Aux blasmes de leur vie, aux hontes de leur mort» (v. 1124-1128 et 1143-1152).

${ }^{5}$ Sur ce point, voir J. Nassichuk, «Jodelle et l'Iliade: la critique d'Homère dans les Discours de Jules Cesar», Plaisir de l'épopée, G. Mathieu-Castellani éd., P.U. Vincennes, 2000, p. 211-228.
} 
au texte, mais cette figure est polyvalente, et sert moins de modèle pour le prince, que de point de vue pour le poète ; et c'est par le biais de cette dernière identification, plus inattendue, que se révèlent le mieux les enjeux politiques de la figure de César.

À la fin de ce qu'on peut, dans un premier temps, considérer comme le prologue du poème ${ }^{6}$, Jodelle annonce le plan de son texte, et la logique de son développement. Après avoir brièvement évoqué la situation alarmante du royaume, Jodelle déclare au roi, son destinataire:

Cela donc me fait poindre en ces pensers divers

D'un prompt et chaud humeur, pour vouloir dans ces vers

De ce Cesar pensif les mesmes discours faire,

Qu'il fit sur tel passage, et pour, et au contraire,

Ausquels je brusle apres d'accommoder les tiens :

Mais premier permets, SIRE, ici chanter les siens ${ }^{7}$.

Il s'agit donc de présenter au roi les méditations de César avant le passage du Rubicon, avant de les «accommoder » à la conjoncture à laquelle il est confronté. La pertinence de cet exercice d'application tient au fait que les deux personnages sont dans une situation analogue. Au roi, Jodelle déclarait, quelques vers auparavant:

Car je sen que desja la rage turbulente

De ce siecle, bien tost à passer te presente

Maint nouveau Rubicon ${ }^{8}$.

Cette analogie permet de déduire la date du texte : si le roi est au bord du Rubicon, c'est que les guerres de religion sont sur le point d'éclater, et que l'armée royale est prête à partir en guerre contre les protestants. Cette situation renvoie au printemps de 1562 , entre le massacre de Vassy ( $1^{\mathrm{er}}$ mars) et la prise d'Orléans par les protestants ( 2 avril) $)^{9}$. Si l'édit de Saint-Germain, du 17 janvier 1562, couronnant une année de négociations, a pu laisser espérer que le conflit pourrait être évité, le massacre de Vassy au printemps a dissipé cette illusion, et, dès lors, Jodelle pouvait sentir (voir «Car je sen»), sans être devin, que le roi aurait bientôt à franchir son Rubicon. À cette date, le roi, Charles IX n'a que 12 ans, et c'est une des fonctions

${ }^{6}$ Si ce développement peut d'abord passer pour un prologue, c'est précisément qu'il se clôt sur l'annonce du plan du poème, et qu'aussitôt après Jodelle évoque César: on a alors l'impression que le poème commence vraiment. Or, nous verrons que l'annonce de plan est équivoque, que César est abandonné peu après avoir été évoqué, et que, dès le prétendu prologue, Jodelle met en place des éléments fondamentaux pour l'interprétation de son entreprise. Bref, le poème commence vraiment dès le premier vers, et ce prologue illusoire est un trompe-l'œil.

${ }^{7}$ V. $225-230, O M P$, fol. $139^{\mathrm{r}}$; O.C. II, p. 298.

${ }^{8}$ V. 219-221, ibid.

${ }^{9}$ Balmas envisage une période de composition un peu plus large, entre janvier 1561 et mars 1562, voir O.C. II, p. 479. En tout cas, d'autres éléments biographiques incitent à dater ce poème d'avant 1563 , voir infra, n. 41 . 
du prologue que de justifier cette adresse à un roi enfant. Le magnanime, explique Jodelle, accepte de servir, librement et à distance, le prince dont il a reconnu la vertu ; et il sert alors

D'un grand cœur, qui n'a point d'eguillon que soymesme,

Celuy que son vouloir prend pour sujet gaillard ${ }^{10}$.

Il s'agit certes d'affirmer la liberté du magnanime, qui n'obéit qu'à son «vouloir » à l'instant même où il se soumet, le prince qu'il se reconnaît fournissant seulement un «sujet» légitime à son désir de service; mais ce paradoxe se comprend d'autant mieux qu'on considère que le roi auquel Jodelle se rallie n'est qu'un enfant, et qu'il n'a encore accompli aucune action propre à révéler sa vertu, et à forcer l'admiration du « grand cœur ».

Car ce que j'ay conceu dedans moy d'esperance,

Des traits que j'ay merquez dès ta premiere enfance,

M'ont fait, sans à ta suite autrement m'asservir,

Comme il t'apparoistra, d'un grand cœur te servir' ${ }^{11}$.

Pour justifier sa dédicace, Jodelle invoque l'«esperance » qu'a fait naître la «premiere enfance» de son dédicataire : c'est que Charles IX n'a encore aucune action éclatante à son crédit, et qu'en louant sa vertu, c'est-à-dire sa puissance d'accomplir de hauts faits, Jodelle peut le louer d'actions encore à venir' ${ }^{12}$. La reconnaissance du mérite est un argument qui garantit au magnanime l'indépendance, le désintéressement de son éloge, et qui permet à Jodelle de louer un roi qui n'a encore rien accompli. En outre, la vertu latente du jeune roi justifie aussi l'analogie avec César, qui possédait la puissance d'accomplir de grandes choses, si bien que ce thème de la vertu royale justifie la démarche exemplaire de Jodelle.

Il est possible que cette démarche soit orientée par la méthode machiavélienne, telle qu'elle apparaît en particulier dans les Discours sur la première décade de Tite-Live: nous rencontrerons en tout cas cette référence à plusieurs reprises. En effet, Machiavel y scrute l'histoire romaine, dans une démarche de comparaison avec les événements de l'actualité, et il tente d'en dégager des lois historiques, propres à éclairer l'action présente. Il ne s'agit pas à proprement parler d'imiter un modèle antique, mais plutôt d'analyser les causes d'un épisode du passé, pour orienter l'action présente ${ }^{13}$. Jodelle part de même de l'exemple de César au bord du Rubicon, puis analyse l'état de la république romaine avant d'en extraire les «naturelles

\footnotetext{
${ }^{10}$ V. $128-129$, OMP, fol. $137^{\mathrm{v}}$; O.C. II, p. 296.

${ }^{11}$ V. 167-170, OMP, fol. $138^{\mathrm{r}}$; O.C. II, p. 297.

${ }^{12} \mathrm{C}$ 'est bien parce qu'il écrit sous un roi enfant que, dès le début du texte, alors qu'il n'a pas encore établi le parallèle entre le comportement du « grand cœur» et sa propre attitude, Jodelle note que le magnanime ne peut pas se soumettre à un prince «sans qu'un eguillon que luy peut faire naistre / La Vertu, pour prevoir l'honneur futur d'un Roy» (v. 23-24; OMP, fol. $135^{\mathrm{v}}-136^{\mathrm{r}} ;$ O.C. II, p. 294) : cette référence à «l'honneur futur» suppose un roi qui n'a pas encore fait ses preuves.

${ }^{13}$ Voir G. Colonna d'Istria et R. Frapet, l'Art politique chez Machiavel, Paris, Vrin, 1980, en part. le ch. $1:$ : L'Art de la comparaison ».
} 
loix » qui président au destin des empires ${ }^{14}$ : ces lois vont évidemment peser sur la décision que, dans la partie manquante du poème, le poète aurait incité le jeune roi à prendre, mais rien ne garantit que cette décision aurait été identique à celle de César. Au début du poème, Jodelle souligne que, quand le magnanime veut servir son roi, il va
L'encourageant, s'il peut, aux choses les plus hautes, Des plus grands anciens luy proposant les fautes,
Vertus, ruses, discours, et ce dont la grandeur
Peut renverser, ou croistre, ou sauver son grand heur $[\ldots]^{15}$.

Ces lignes définissent bien entendu le projet de Jodelle lui-même dans les Discours. César compte donc au nombre «des plus grands anciens», mais cette «grandeur » n'exclut ni «fautes » ni « ruses », et dans l'histoire du général romain, le roi de France doit discerner ce qui peut «renverser» son empire, autant que ce qui peut le préserver. Loin d'incarner une vertu ou une grandeur indépassable, César représente un accomplissement remarquable mais imparfait; et si son exemple est proposé au roi de France, c'est pour qu'après l'avoir analysé, celui-ci évite ses erreurs, et ainsi le surpasse. Ce n'est donc pas seulement en fonction d'une analogie de situation que Jodelle soumet le cas de César à Charles IX : entre les deux princes, il y a aussi, ou du moins il devrait y avoir, une communauté de préoccupation, une même tension vers la grandeur. Le général romain constitue moins un modèle pour le roi de France, qu'il définit un paradigme, une famille typologique, dans laquelle le poète veut faire entrer son roi, en même temps qu'il veut l'inciter à dépasser son prototype romain.

La logique exemplaire annoncée est d'ores et déjà perturbée, car le cas antique n'est pas proposé en modèle au roi moderne : Jodelle ne cherche pas à représenter César, pour inciter Charles IX à se conformer à ce patron ; mais il entreprend d'analyser son histoire, pour déterminer ce qu'il faut suivre et ce qu'il faut éviter. Correctement interprété, le titre du poème confirme cette orientation intellectuelle. S'il peut d'abord laisser attendre un événement, une harangue prononcée par Césarc'est en ce sens que l'entend Balmas, quand il constate qu'à la fin du texte, «les discours du célèbre capitaine romain n'ont pas encore commencé »- , cette acception de «discours » comme ; «harangue, paroles prononcées », ne va pas de soi au $\mathrm{XVI}^{\mathrm{e}}$ siècle Dans le cadre du poème de Jodelle, deux autres interprétations s'imposent.

Puisque le mot apparaît dans le titre du poème, il peut renvoyer, non pas au contenu du texte mais à son genre. Dans ce cas, le complément de nom «de Jules Cesar» ne constituerait pas un génitif (les discours qu'a prononcés Jules César), mais un ablatif (les discours à propos de Jules César). La table des matières des Euvres et meslanges poetiques soutient cette lecture. Les poèmes sont en effet classés par genres (sonnets, contr'Amours, chansons, chapitres, etc), et on y trouve une entrée «Discours », sous laquelle deux poèmes sont rangés :

\footnotetext{
${ }^{14}$ L'expression «naturelles loix » se trouve au v. 373, et l'examen des lois qui régissent le cours des empires occupe les v. 343-412, OMP; fol. $141^{\mathrm{r}}-142^{\mathrm{v}} ;$ O.C. II, p. 301-302.

${ }^{15}$ V. 143-145; OMP. fol. $138^{\mathrm{r}}$; O.C. II, p. 296.
} 


\author{
De Jules Cesar 135.b \\ Contre la Riere-venus 65. $\mathrm{a}^{16}$
}

Dès lors, ces «Discours » se confondent avec le poème que nous lisons; ils ne sont pas tenus par César, mais par le locuteur lui-même, et il est vain d'en chercher le commencement dans le texte, puisqu'ils commencent dès le premier vers. On peut être tenté de rapporter cet usage générique du mot «discours » dans le titre d'un poème à l'exemple du Discours des miseres de ce temps, de Ronsard. Sans doute même les éditeurs de Jodelle, qui ont établi la table de l'édition posthume de ses œuvres, ont-ils eu cette référence en tête, car cette table révèle par ailleurs le désir d'organiser les Euvres et meslanges poétiques de Jodelle sur le modèle des Euvres de Ronsard, en reprenant les mêmes catégories génériques ${ }^{17}$. Cependant, le projet des Discours de Jodelle n'a pas grand chose à voir avec celui de Ronsard et, si notre hypothèse de datation est correcte, les Discours de Jodelle sont antérieurs ${ }^{18}$. Dans l'acception générique que suggère la table, le mot «discours » devrait être au singulier ${ }^{19}$, alors que le titre du poème le donne sans ambiguïté au pluriel : «Les discours de Jules César». Au-delà de la référence ronsardienne, fruit d'une réinterprétation générique des éditeurs, on peut alors envisager un autre modèle pour le titre de Jodelle, celui des Discours de Machiave ${ }^{20}$. Nous avons signalé des analogies entre ces deux auteurs, et nous rencontrerons d'autres points de contact entre leurs textes : la référence générique est donc possible, et elle présente l'intérêt de donner une acception très intellectuelle au mot «discours ».

$\mathrm{Si}$ on se tourne maintenant vers le corps du texte lui-même, il faut constater qu'à plusieurs reprises, Jodelle explicite son titre dans le sens intellectuel que nous avons commencé à dégager. Ainsi, lorsqu'il présente l'argument de son poème, il déclare vouloir

\footnotetext{
${ }^{16} O M P$. sig. II iv v.

${ }^{17}$ Voir nos remarques dans l'introduction de : É. Jodelle, Les Amours. Contr'Amours. Contre la Riere-Venus, éd. E. Buron, P.U. Saint-Étienne, 2003, p. 7-8.

${ }^{18}$ Selon Paul Laumonier, dans son édition de Ronsard (t. XI, p. XIV), le «Discours des miseres de ce temps » a été publié en mai 1562, alors que nous avons daté supra les Discours de Jodelle d'avant le 8 avril.

${ }^{19}$ C'est le cas pour Ronsard, comme l'indique, non pas le Discours lui même, mais la Continuation $d u$ - et non «des »-Discours des miseres de ce temps, sans doute composée vers le $1^{\text {er }}$ octobre 1562 (voir Laumonier, loc. cit. p. XV). Le mot «discours » n'apparaît au pluriel que dans le titre du tome VI - «Les discours »- de l'édition des Euvres de Ronsard de 1567 ; mais il désigne alors, non pas un poème, mais le recueil des textes que Ronsard a écrit contre les protestants.

${ }^{20}$ Jodelle connaissait Le Prince, puisqu'il a écrit des vers liminaires pour la traduction française de cet ouvrage procurée par Guillaume Cappel en 1553 ; et il était ami de Jacques Gohory, qui a traduit les Discours de Machiavel en 1544 et en 1548 : Le Premier livre des discours de l'estat de paix et de guerre, de Messire Nicolas Macchivegli, Secretaire et citoyen Florentin, Sur la premiere decade de Tite-Live, Paris, D. Janot, 1544. Voir E. Balmas, «Jacques Gohory, traduttore del Machiavelli », Studi Machiavelliani, Vérone, Pallazzo Giuliari, 1972, p. 3-52 ; repris dans saggi e studi sulla letteratura francese, Padoue, Liviana, 1982, p. 23-73.
} 
De ce Cesar pensif les mesmes discours faire :

Qu'il fit sur tel passage, et pour, et au contraire ${ }^{21}$.

En relation avec l'adjectif «pensif», le nom de « discours» reçoit son acception la plus courante au $\mathrm{XVI}^{\mathrm{e}}$ siècle, celle de : pensée, méditation. Il faut prêter une attention scrupuleuse à la syntaxe, quand Jodelle écrit qu'il veut «dans ces vers / De ce Cesar pensifs les mesmes discours faire». «Ce Cesar» est bien introduit par la préposition «de», et non par la conjonction «que». Jodelle ne veut donc pas faire les mêmes discours que César, mais bien faire les discours mêmes de César, où l'adjectif «mesmes» signale l'identité entre la pensée du poète et celle du général. Les «discours» que développent le poète sont bien ceux de Jodelle, mais ces pensées coïncident exactement avec celles de César, qu'elles réactualisent. D'ailleurs, Jodelle déclare un peu auparavant.

Tant qu'il ne tourne en moy gueres moins de pensees,

Que Cesar en sentit dedans soy r'amassees,

La nuict dont il vouloit passer le lendemain

Le Rubicon ${ }^{22} \ldots$

Les «discours » de César correspondent à ces «pensées » qui «tournent » en lui ; ce qui confirme à la fois le sens intellectuel du mot «discours », et l'analogie entre les pensées du poète et celle du général.

Le titre est donc à triple entente : les Discours de Jules César sont tout à la fois ceux que développe le général romain, ceux que développe Jodelle à propos du général romain, et, puisque ces «discours » se confondent et coïncident avec le texte, le mot «discours» désigne aussi le texte lui-même, et définit son genre. Bref, il s'agit, pour Jodelle, de reconstituer les méditations de Jules César, mais en focalisation externe, sans faire parler ce personnage à la première personne, mais en reconstituant, par un phénomène d'empathie poétique, la réflexion qui a été la sienne.

Le débat sur la meilleure forme de constitution permet d'apprécier précisément comment s'opère cette superposition de points de vue. Cette discussion vise à réprouver le régime démocratique, car l'égalité sur laquelle il se fonde est, par définition, inconciliable avec la reconnaissance de la grandeur. Ce développement constitue apparemment une digression, comme si Jodelle oubliait César pour exposer ses propres vues politiques; pourtant cette analyse acquiert une pertinence indéniable quand on la rapporte à César au moment de passer le Rubicon : en effet, le général s'apprête à renverser la république romaine, régime qui, s'il n'est pas démocratique, n'en est pas moins collectif, et opposé à la royauté. C'est donc contre les «premiers de Rome », soucieux de conserver leur position, et inquiets autant qu'envieux de sa grandeur, que César doit imposer sa prééminence. La réflexion politique de Jodelle, sur la grandeur en régime démocratique, amplifie donc les méditations possibles de César sur le bord du Rubicon, au moment d'entrer en guerre contre son propre pays pour lui imposer son autorité.

${ }^{21}$ V. 227-228, OMP, fol. $139^{\mathrm{r}}$; O.C. II, p. 298.

${ }^{22}$ V. 207-210, ibid. 
Le jeu des allusions confirme cette analogie des points de vue. En effet, quand Jodelle discute les trois formes de régime, il les illustre par des allusions aux institutions de la république romaine. Ainsi quand il évoque «une Aristocratique Façon de gouverner quelque grand république», il en rapporte la conduite aux «hauts et saincts decrets d'un Senat ${ }^{23}$. Dans l'analyse des institutions de la république romaine que propose Polybe, source fondamentale de la réflexion constitutionnelle au $\mathrm{XVI}^{\mathrm{e}}$ siècle ${ }^{24}$, la longévité exceptionnelle de ce régime est attribuée au fait qu'il réussit à fusionner les trois types de constitution : monarchique, aristocratique et démocratique ${ }^{25}$. Dans cet équilibre, l'autorité du Sénat répond à un fonctionnement de type aristocratique, analyse que Jodelle reprend à son compte. De même quand il défend le principe de hiérarchie, il souligne qu'il faut toujours

qu'entre les plus hauts les uns sur tous president,

Voire un seul, ou bien deux, qui prennent presque en soy,

(Le seul nom excepté) tout ce qui est d'un Roy :

Mais leur charge et puissance, ou bien n'est qu'annuelle

Seulement, ou bien n'est qu'autant que les appelle

A cela le besoin, encore leurs actions

Cedent aux loix, et mesme aux superstitions :

Qui plus est, quelquesfois de nouvelle ordonnance

Et de controullemens, se borne leur puissance ${ }^{26}$.

Il décrit principalement la fonction des consuls : élus à deux, pour un an, ils jouissent de l'imperium, du haut commandement sur les opérations militaires, si bien que Polybe estime leur pouvoir quasi royal, sauf que la durée de leur mandat est déterminée par la loi et que, pour toute décision financière, ils sont soumis au Sénat, qui exerce ainsi un contrôle («controullemens») sur leur décision. Jodelle évoque encore un magistrat de puissance royale, unique («un seul, ou bien deux») et désigné, non pour un an, mais «autant que les appelle / à cela le besoin »: sans doute s'agit-il du dictateur (fonction dont Polybe ne parle pas). Enfin, une trentaine de vers plus bas, ce sont les tribuns du peuple que Jodelle évoque, quand il note que, dans la république romaine, rien ne se peut faire

Sans un accord de tous, fust-ce du populaire,

Qui puissant en l'estat (bien qu'il soit le plus bas)

Ha pour cela ses voix, et propres magistrats,

Dont l'authorité mesme à toute autre s'oppose,

Tirant souvent à soy pour la publique chose

Tout vueil, et tout pouvoir des armes, et des loix,

Tant il craint que les grands facent sur luy les $\operatorname{Rois}^{27}$.

${ }^{23}$ V. 455-457, OMP, fol. $143^{\mathrm{r}} ;$ O.C. II, p. 303..

${ }^{24}$ Sur l'importance de l'analyse de Polybe, et sa remise en question par Bodin, voir J. Franklin, Jean Bodin et la naissance de la théorie absolutiste, Paris, PUF, 1993, p. 52-54; sur l'idéal politique qu'il soutient, voir A. Jouanna, «Un idéal d'ordre politique en France au $\mathrm{XVI}^{\mathrm{e}}$ siècle : la monarchie mixte », Ordre et désordre dans la civilisation de la Renaissance, G. Pérouse et F. Goyet éd., P.U. Saint-Étienne, 1996, p. 51-63.

${ }^{25}$ Voir Polybe, Histoires, 1. 6, 11-18.

${ }^{26}$ V. 468-476, OMP, 143 r-v ; O.C. II, p. 303-304 
Bref, la discussion sur les différentes formes de gouvernement se fonde sur les institutions de la république romaine : Jodelle appuie son analyse sur une réalité politique que César pouvait connaître, afin de reconstituer son discours.

Simultanément, le poète insère dans sa réflexion des allusions contemporaines. Ainsi, lorsqu'il laisse éclater son mépris contre «l'estat tout populaire » ou règne «un populaire vil», il évoque le malheur du magnanime contraint de vivre

sous quelque gros Canton

De Suisses, Grisons, ou bien d'autres sauvages,

En leur joug tant ignoble avilissans leurs âges.

Que cent fois soyent maudits (si lon dit vray) tous ceux,

Qui entre nous vouloyent tâcher nous faire à eux

Semblables, en estat: Grande estoit leur furie

Hypocrite, plus grande encor leur barbarie ${ }^{28}$.

Au-delà des Suisses, ces vers maudissent les mutins d'Amboise («ceux qui, entre nous...»), que la rumeur catholique accusait de vouloir renverser la monarchie française pour instaurer l'état populaire en France. Régnier de La Planche note que, dès que les Guises ont été mis en cause par les mutins, ils ont fait courir le bruit que les protestants voulaient éliminer la famille royale "pour plus à l'aise abolir toute authorité royale, réduire le royaume en cantons et république, tuer toute la noblesse de France, à l'exemple des Suysses, pour vivre en commun ${ }^{29}$. P. de La Place rapporte la même rumeur : les protestants avaient pour but «d'abbatre la monarchie de France, et la réduire en forme de république et estat populaire, tout ainsi qu'est le pays de Suisse $»^{30}$. De même que les points de vue du poète et de César se confondent, les références se partagent entre leurs domaines d'expérience respectifs.

Jodelle a peut-être trouvé le cadre de sa reconstitution des pensées de César dans quelques indications de Plutarque, qui note qu'à l'approche du Rubicon, le général «s'arrêta tout coi » et sentit

en l'esprit un remords de penser à ce qu'il attentait ; et plus il variait en ses pensements, quand il considérait la grande hardiesse de ce qu'il entreprenait. Si fit adonc plusieurs discours en son entendement sans en dire mot à personne, inclinant tantôt en une part et tantôt en une autre, et changea son conseil en beaucoup de partis contraires à part soi ; aussi en disputa-t-il beaucoup avec ceux qu'il avait de ses amis quant et lui ${ }^{31}$.

${ }^{27}$ V. 500-506; OMP, fol. $143^{\mathrm{v}}-144^{\mathrm{r}} ;$ O.C. II, p. 304.

${ }^{28}$ V. 622-628, OMP, fol $146^{\mathrm{r}} ;$ O.C. II, p. 307.

${ }^{29} \mathrm{R}$. de La Planche, Commentaires de l'estat de la religion..., in J.A.C. Buchon, Choix de chroniques et mémoires sur l'histoire de France (XVIe siècle), Paris, A. Desrez, 1836, ibid., p. 37, col. 1.

${ }^{30}$ P. de La Place, Histoire de l'estat de France..., ibid., p. 255 col. 2.

${ }^{31}$ Plutarque, Les Vies des hommes illustres, trad. J. Amyot, éd. G. Walter, Paris, Gallimard, 1951, t. II, p. 448-449. Jodelle était un lecteur des Vies, puisqu'en 1553, six ans avant la traduction par Amyot, il s'est inspiré de la «Vie d'Antoine» pour écrire Cleopatre captive 
Plus qu'Appien ou Lucain, qui évoquent aussi l'arrêt méditatif du général sur le bord de la rivière, Plutarque insiste sur la perplexité de César, en des termes proches de ceux de Jodelle. Le mot «discours» apparaît dans ce passage, et il est employé comme synonyme de "pensements»; et l'effet de ces «discours» est d' «inclin[er] tantôt en une part et tantôt en une autre »: Jodelle note de même que ses pensées tumultueuses l'entraînent «tantost devers ceci, tantost devers cela $»^{32}$. Plus bas, il ajoute qu'il veut reconstituer les discours de César «et pour, et au contraire» (v. 228, cité supra). Dans les deux cas, le même mot, «discours », se traduit par un balancement («tantôt... tantôt...»; « et... et...»), signe de la perplexité du général, dans une délibération douteuse. Pour un auteur comme Jodelle, peu enclin à l'imitation, le grand intérêt de Plutarque tient au fait qu'il constitue une source fort peu contraignante. Il signale que les premières méditations de César furent muettes, et il n'en dit rien d'autre: "Si fit adonc plusieurs discours en son entendement sans en dire mot à personne». Plutarque ajoute aussitôt après que le général débattait avec ses proches

de combien de maux par le monde serait cause et commencement ce passage de la rivière, et combien leurs successeurs et survivants en parleraient un jour à l'avenir ${ }^{33}$.

Ces lignes esquissent seulement un cadre problématique, et n'orientent qu' $a$ minima l'invention de Jodelle, qui a toute liberté pour les développer à son gré. De fait, le plan des Discours suit ces indications schématiques. César envisage les conséquences immédiates du franchissement de la rivière, ainsi que la mémoire que ce fait laissera dans la postérité. Or, la première moitié des discours développe différentes questions politiques à partir de l'examen de la république romaine, et elle amplifie la réflexion de César sur les conséquences intérieures du passage du Rubicon ; et la seconde moitié porte sur la partialité de la postérité. Même si les exemples nous éloignent de César, le sujet même de cette réflexion nous renvoie à ses préoccupations, au moment où il joue sa renommée posthume - passera-t-il pour le fondateur de l'Empire ou pour un factieux ? - sur le coup de dé du Rubicon. Ainsi, Les Discours amplifient les indications de Plutarque ; le poème se modèle sur la pensée du général romain : il ne déborde pas le cadre des préoccupations de celui-ci, et sa construction digressive répond à l'agitation tumultueuse de ses pensées. En d'autres termes, la figure de César fonctionne comme principe régulateur du développement du poème.

Les « discours » de César coïncident donc parfaitement avec ceux de Jodelle, c'est-à-dire avec le poème lui-même. César devient ainsi une figure de l'auteur, et c'est ce mouvement qui perturbe le plus radicalement la logique de l'exemple annoncée au début du texte : comme Jodelle se projette dans la figure du général romain, c'est son propre «discours » qu'il propose comme une règle de conduite au jeune roi, et c'est grâce à lui qu'il entend orienter l'action royale. En s'identifiant à

(voir M. Delcourt, « Jodelle et Plutarque », Bulletin de l'Association Guillaume Budé, janvier

1934, p. 36-52).

${ }^{32}$ V. 214, OMP , fol. $139^{\text {r }}$; O.C. II, p. 298.

${ }^{33}$ Plutarque, loc. cit. 
César, Jodelle revendique en somme, de façon à peine voilée, un rôle de conseiller. Toutefois, le choix d'une telle figure d'auteur ne s'épuise pas dans la mise en évidence de l'intention stratégique qui la soutient: elle engage en fait toute l'éthique poétique de Jodelle.

L'identification du poète à César est explicitement posée dans les Discours, juste avant que Jodelle propose le général romain en modèle au roi, et pour en comprendre les enjeux, il faut la réinscrire dans le développement du poème. Les Discours commencent par une longue réflexion sur la manière dont les «mieux nés " (v. 2) doivent s'acquitter du « service » et de la « suite» des grands : à distance, car ils sont impatients de tout «service servile» (v. 39). Sans grande surprise, Jodelle s'identifie à ces magnanimes en retrait ${ }^{34}$, mais les Discours de Jules Cesar marquent la fin de cette période de service obscur: Jodelle adresse son poème à Charles IX pour faire reconnaître ses services antérieurs, et pour le servir maintenant au grand jour ${ }^{35}$. Il est donc sur le point d'imposer à sa carrière un tournant majeur, et, après avoir servi le roi par «un domestic / Service recelé», il se prépare à lui en rendre un «public». C'est à ce moment qu'il se compare à César, prêt à franchir le Rubicon :

Je resen bien, mais c'est pour dissemblable chose,

Qu'un estroit Rubicon à passer se propose,

A moy comme à Cesar. [...]

Tant qu'il ne tourne en moy gueres moins de pensees,

Que Cesar en sentit dedans soy r'amassees,

La nuict dont il vouloit passer le lendemain

Le Rubicon, pour faire à son pays Romain

La guerre, et de fureur juste ensemble et inique,

Le ventre maternel de sa grand Republique,

Parricide fouler ${ }^{36}$.

Cette étonnante analogie entre le poète et César devant le Rubicon se fonde sur le fait qu'ils sont tous deux sur le point de prendre une décision audacieuse, qui engage la suite de leur carrière. Alea jacta est: Jodelle pourrait reprendre à son compte la formule de César. Plus précisément, tous deux préparent leur irruption dans la vie publique de leur temps : franchissant le Rubicon, César revient de Gaule en Italie, et entend faire reconnaître à Rome l'autorité qu'il a conquise à l'étranger. De même Jodelle sort de sa retraite pour révéler ses écrits au roi, et éventuellement au royaume, consacrant ainsi ses écrits au public et au bien public. Le point le plus troublant de l'analogie, c'est que, dans le cas de César, ce surgissement dans l'espace de la cité répond à la décision d'entrer en guerre civile; et que Jodelle fait sien aussi cet aspect ambigu de la décision du général. Après avoir déclaré qu'il allait franchir son Rubicon, il poursuit :

\footnotetext{
${ }^{34}$ «Or tout ceci m'avient, qui hors de ta presence / T'ay choisi pour mon but, te servant en absence », v. 163-164, OMP, fol. $138^{\text {r }}$ O.C. II, p. 297.

${ }^{35}$ «Le temps veut commencer, sans que je vueille dire / Ici qu'il en est, à te decouvrir, SIRE,/ Quel service est le mien : voulant faire avancer / Devers toy mes labeurs, et me faut commencer / Par une arre petite... », v. 179-183, OMP, fol. 138v ; O.C. II, p. 297.

${ }^{36}$ V. $195-197$ et $207-212$; OMP, fol. $138^{\mathrm{v}}-139^{\mathrm{r}}$; O.C. II, p. $297-298$.
} 


\begin{abstract}
Car pour estre incogneu
Jusqu'ici, je sçay bien quel grand heur m'est venu, Je sçay bien, veu le temps, qui contre nostre teste Nous reforge sans fin divers traits de tempeste, Que s'il peut bien sçavoir, ce que sur luy je puis, Ce m'est d'estre cogneu pour tout tel que je suis, Un grand malheur, peut estre, et continuel trouble: Si tu n'as, SIRE, en main le bouclier sept fois double, Dont un Ajax de gloire et de fureur ardent,

En combatant couvroit Ulysse le prudent ${ }^{37}$.
\end{abstract}

Il y a «grand heur » à « estre incogneu », et «grand malheur» à devenir «cogneu : se rendant public, le poète s'expose aux attaques de ses contemporains, et la comparaison avec Ulysse suggère qu'il ne peut, seul, en triompher. Au livre XI de l'Iliade en effet (v. 472-488), Ulysse est sur le point d'être surmonté par ses adversaires troyens, mais il est sauvé par l'intervention de Ménélas et d'Ajax, ce dernier le protégeant de son bouclier (v. 485-486). Au delà du fait qu'elle introduit le motif de la guerre de Troie, et la référence à l'Iliade, que Jodelle discutera longuement à la fin du poème, cette comparaison entre le poète et Ulysse a pour fonction d'appeler sur le poète la puissante protection du roi, mais elle n'en représente pas moins le rapport du poète au public, qu'il affronte en sortant de sa retraite, comme un combat. Qu'il soit en passe de perdre ce combat n'enlève rien au fait que la sortie en public correspond, comme dans le cas de César, au déclenchement d'une guerre civile.

Pour comprendre cette analogie, il faut faire retour sur la carrière de Jodelle au cours des années 1550. Au début de cette décennie, qui coïncide aussi avec le début de sa carrière, Jodelle refuse de publier ses vers, convaincu que la France est en proie à l'envie, vice national qui la conduit à se mutiner contre les grands hommes qui pourraient l'honorer, à souhaiter la mort de ces champions qui, par leur gloire, l'humilie. Vers le milieu de la décennie, Jodelle entre au service de Marguerite de France, la sœur du roi Henri II, ce qui l'oblige à modérer son refus de publication, qu'il annonce maintenant prochaine, mais diffère sans cesse ; néanmoins, le poète ne tempère pas l'amertume de son jugement sur le royaume. En 1556, il écrit une épître à Marguerite de France, liminaire pour le second livre des Hymnes de Ronsard, qui synthétise sa position d'alors. Il s'emporte toujours contre les français,

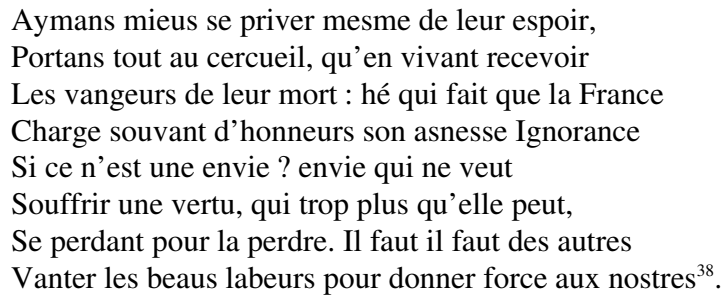

${ }^{37}$ V. 197-206 ; OMP. fol, $138^{\mathrm{v}}-139^{\mathrm{r}}$; O.C. II, p. 297-298. 
Cette diatribe contre le royaume dessine en creux l'éthique du poète magnanime, qui doit s'employer à favoriser les vertueux, et à les faire reconnaître. Jusqu'à ce point, ce credo rejoint celui que Jodelle déclinait auparavant. La nouveauté tient au fait qu'il envisage maintenant la publication. À Marguerite, il déclare en effet :

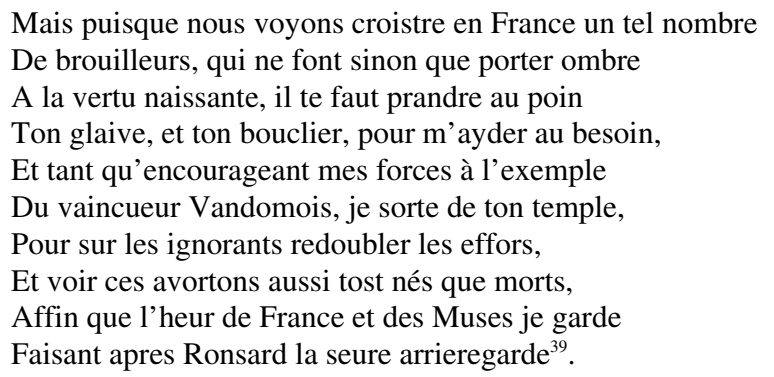

Ayant accédé à la protection de la princesse, le poète doit maintenant publier ses œuvres, mais il conçoit toujours cette publication sur le mode du combat, d'une guerre contre l'ignorance et l'envie, dans laquelle le grand homme aura toujours le dessous, s'il n'est pas protégé par le bouclier que constitue la protection d'un prince. L'image est la même ici que dans les Discours, où le bouclier d'Ajax protégeant Ulysse se substitue à l'Égide de Pallas. Publier, c'est bien entrer en guerre contre son pays : le lien avec le franchissement du Rubicon devient alors évident ${ }^{40}$. Pour compléter cette contextualisation des Discours, il faut encore ajouter que, pour des raisons inconnues, Jodelle a été condamné à mort en 1558, au lendemain de l'organisation d'une mascarade qui a tourné au fiasco. Le poète s'est enfui, et il semble avoir été exécuté en effigie. En 1562, il n'a pas encore rejoint la cour, et, à l'évidence, la composition des Discours pour Charles IX vise à reconquérir une partie de sa faveur perdue ${ }^{41}$. Ces circonstances dramatisent encore un peu plus

${ }^{38}$ O.C. I, p. 100

${ }^{39}$ Ibid., p. 101.

${ }^{40} \mathrm{Au}$ début des Tragiques, Agrippa d'Aubigné s'identifie à César sur le point de franchir le Rubicon, par le biais d'une réécriture de Lucain (Les Tragiques, éd. J.R. Fanlo, Paris, Champion, 1995, t. I, p. 50-53, v. 7-19), et il assimile ainsi l'amorce du poème à une entrée en guerre civile. Étant donné l'admiration qu'il portait à Jodelle, on peut lire ces premiers vers des Tragiques comme la réécriture d'un motif essentiel des Discours de Jules Cesar. Avec un déplacement important: Jodelle associe le passage du Rubicon à la publication de ses textes, alors qu' Aubigné l'assimile au commencement du poème. Sur la relation d'Aubigné à Jodelle, sur la manière dont il fonde son éthique auctoriale et dont il légitime sa pratique éditoriale sur le refus jodellien de la publication, voir notre article: "Aubigné et Jodelle. Genèse d'une fiction énonciative", à paraître dans les actes du colloque «Fiction et histoire dans l'œuvre d'Agrippa d'Aubigné » (Genève, décembre 2002).

${ }^{41}$ En revanche, divers poèmes laissent penser que le poète a retrouvé la faveur royale dans la première moitié de 1563 , au plus tard en juillet. Voir la suite de trois «sonnets. A la Royne mere » $\left(O M P\right.$, fol. $187^{\mathrm{r}-\mathrm{v}} ;$ O O.C.. I, p. 204-205, s. I-III) . Dans sa notice, Balmas date ces trois textes du lendemain de la reconquête du Havre, le 17 juillet 1563 , mais cette date ne vaut que 
l'antagonisme entre le poète et le royaume, et permettent de comprendre pourquoi, au début des Discours, Jodelle se présente comme un grand cœur fuyant la cour, servant fidèlement son prince, mais à distance.

Dans les Discours, Jodelle ne se contente pas de reproduire cette analyse de l'antagonisme entre le magnanime et son siècle: il tire les conséquences politiques de l'idéologie de la grandeur qu'elle implique. Les arts et les lettres ne lui paraissent possibles que dans une société hiérarchisée, inégalitaire.

Aussi croire il nous faut que d'une multitude,

Sans quelques nobles chefs l'estat est vil, et rude,

Incertain, confus, lâche, ignoble, et qui ne peut

Avoir l'honneur en soy, qui seul pourtant nous meut

Non seulement aux faits, qui par l'heur de la guerre,

Du nom, du los, du bien, font l'accroissance acquerre :

Mais aux vertus, aux arts, aux sciences aussi,

Bref, à tout ce qu'on peut cognoistre et suivre ici

De bon, de beau, de grand, et sans qui (je croy) qu'estre

Seroit pis que mourir, ou bien jamais ne naistre $[\ldots]^{42}$. conclut :

Puis, après avoir détaillé les dons requis pour les arts et sciences, Jodelle

Or quiconques dans soy tous ces dons goustera,

D'un populaire vil sans fin dedaignera

L'estat tout populaire : et n'y a rien qui blesse

Un noble esprit, si fort, que de voir sans noblesse

Tous ceux entre lesquels, comme un astre qui luit

Un peu, mais tout autour couvert de noire nuict,

Il luy convient trainer indignement sa vie $[\ldots]^{43}$.

La démocratie, l'état populaire, est impropre aux arts : un «noble esprit » ne peut s'épanouir que dans la noblesse, ce qui suppose une inégalité sociale, et détermine les formes de gouvernement acceptables ou non. On retrouve ainsi les valeur qui inspiraient la comparaison des différentes constitutions. Magnanimité poétique et magnanimité politique sont donc liées : une même éthique de la grandeur établit une parenté entre les causes du poète et du prince, et justifie le rapport conflictuel avec son propre pays.

La première conséquence de cette comparaison du poète avec César réside dans une interprétation positive de la guerre civile déclenchée par le franchissement du Rubicon. Puisque l'espace public est le lieu où se déchaîne la haine envieuse des médiocres, la guerre civile apparaît comme la lutte nécessaire à la restauration de la grandeur. Tel est bien l'enjeu du long développement que Jodelle consacre à la dé-

pour le dernier sonnet, qui contient seul une allusion à cet événement : on peut toutefois établir que les deux premiers sonnets sont probablement antérieurs.

${ }^{42}$ V. 515-524, OMP, fol. $144^{\mathrm{r}}$; O.C. II, p. 304-305.

${ }^{43}$ V. 613-619, OMP, fol. $145^{\mathrm{v}}$; O.C. II, p. 307. 
composition de la république romaine. Il constate d'abord que l'impressionnante série de victoires de César en Gaule ne «grevoit» pas seulement «les cœurs » gaulois, mais

les cœurs des siens aussi

Et sur tout de ceux là qui les premiers de Rome

Se voyoient peu à peu devancer d'un tel homme.

$[\ldots]$

Tant que de grandeur telle en eux ils concevoyent

Crainte, envie, et fureur. ${ }^{44}$

Dans ces vers, Jodelle ramène les antagonismes politiques de la fin de la république à un principe passionnel: l'hostilité des optimates, les «premiers de Rome», envers César est d'abord la conséquence de la jalousie qui les dévore. Jodelle n'envisage leur argumentation politique que dans un second temps, comme une émanation de cette envie fondamentale: la grandeur de César menace la pérennité

De leur hautesse acquise, en un coup terminee

Avec l'estat public, avec la liberté

En qui l'heureux estat sans cesse avoit esté.

Bien que liberté lors ne fust qu'une voix feinte

Et couleur faulse entr'eux. ${ }^{45}$

L'invocation de la liberté n'est qu'un leurre, une transposition politiquement acceptable de la défense des intérêts des dirigeants. Jodelle développe alors un tableau de la liberté romaine agonisante, entourée de «mille feux» dessinant au tour d'elle «telle trainée» (v. 291) qu'il ne fallait que l'intervention d'un homme entreprenant pour tout embraser.

Quand entre les Romains ce Cesar ne seroit

Romme alors pour cela cent Cesars se feroit. ${ }^{46}$

Bref, la liberté était à bout de course, et d'une manière ou d'une autre, son renversement était inévitable. On ne peut donc reprocher à César d'avoir renversé un régime exténué : il s'impose contre la «crainte, envie et fureur» de ses adversaires. Grandeur contre envie : les causes du poète et du prince se confondent.

En somme, on peut considérer que le César de Jodelle procède d'une réinterprétation de l'image politique ou républicaine du général romain, tel qu'il apparaît chez Machiavel par exemple. Le point commun entre ces deux approches, c'est que César est perçu dans son rôle d'adversaire, non seulement de la république romaine, mais aussi de la liberté. Dans cette perspective, Machiavel tient César, malgré sa popularité, pour le premier des tyrans de Rome, celui qui a ouvert la voie des Empe-

${ }^{44}$ V. 253-256 et $269-270, O M P$, fol. $139^{\mathrm{v}}-140^{\mathrm{r}}$; O.C. II, p. 299.

${ }^{45}$ V. $284-288, O M P$, fol. $140^{\mathrm{r}} ;$ O.C. II, p. 299.

${ }^{46}$ V. 341-342, OMP, fol. $141^{\text {r }}$; O.C. II, p. 301. 
reurs, aussi tyranniques que lui, mais moins aimés du peuple ${ }^{47}$. Cette vision négative du personnage n'est pas propre à la Florence républicaine : c'est par exemple celle qu'on retrouve dans le Discours de la servitude volontaire, d'Étienne de la Boétie: «Jules Cæsar [...] donna congé aus lois et à la liberté », et «son humanité mesmes », au nom de laquelle il fut aimé du peuple, ne fut que «venimeuse douceur qui envers le peuple romain sucra la servitude $\aleph^{48}$. Bien que La Boétie s'inspire des Discorsi de Machiavel $^{49}$, l'idéal politique des deux textes ne coïncide pas, car ils ne se font pas la même idée de la «liberté $»^{50}$. Comme le note Ronald Syme, analysant «la révolution romaine », soit le passage de la république à l'Empire :

Libertas est un concept vague et négatif - le mot désigne l'affranchissement de la domination d'un tyran ou d'un parti. Il s'ensuit que libertas, comme regnum ou dominatio, est un terme bien fait pour les duperies politiques. La libertas était le plus ordinairement invoquée pour la défense de l'ordre existant par des individus ou des classes maîtresses du pouvoir et de la fortune ${ }^{51}$.

Le même mot d'ordre peut ainsi servir différentes causes, mais, de Machiavel à la Boétie, César se définit comme celui qui a renversé la liberté. Pour Jodelle également, sauf que, prenant acte du fait que la liberté n'est souvent que le voile d'intérêts particuliers, et qu'à Rome, ce mot d'ordre servait la domination d'optimates mesquins, il justifie l'entreprise de César. Pour éviter que ce rôle d'ennemi de la liberté projette sur lui, par le jeu automatique de l'antonymie, la figure négative du tyran, Jodelle devait placer son action sous le signe d'une valeur susceptible de le justifier positivement. Son César magnanime et adversaire de l'envie est la réfection du César tyran des républicains.

Cette opposition de valeurs, entre liberté et grandeur, correspond aussi à une opposition politique, entre république et monarchie, et, à la veille des guerres civiles, elle donne une légitimité au roi qui s'apprête à entrer en guerre avec une partie de ses sujets. César devient ainsi une figure idéale du roi pendant les guerres de religion. À la fin du siècle, et au début du suivant, l'assimilation d'Henri IV à César deviendra un lieu commun ${ }^{52}$ : c'est qu'il sera clair alors que le roi a effectivement pu refonder un pouvoir monarchique, ce qui relevait d'un pari douteux au cours des quarante années qui ont précédé. Quoi qu'il en soit, et même si c'est de manière plus sporadique et inquiète, cette image de César en fondateur de la monarchie se fait

\footnotetext{
${ }^{47}$ Voir, en particulier, le ch. I. 10 des Discours sur la première décade de Tite-Live.

${ }^{48}$ E. de la Boétie, De la Servitude volontaire ou contr'un, éd. M. Smith -M. Magnien, Genève, Droz, 2001, p. 59-60.

${ }^{49}$ Voir J. Balsamo, «Le plus meschant d'entre eux ne voudroit pas estre Roy » : La Boétie et Machiavel », Montaigne Studies, XI, 1999, p. 5-27.

${ }^{50}$ Sur l'interprétation du texte de La Boétie, voir notre article, «le Discours de la servitude volontaire et son double », Studi francesi, XLV (2001), p. 498-532.

${ }^{51}$ R. Syme, La Révolution romaine, Paris, Gallimard, 1967, p. 152.

${ }^{52}$ Voir G. l'Apostre, La Devise du grand Henri IIII. Roy de France et de Navarre, où il est du tout comparé à César: et les guerres de la ligue raportées de point en poins [sic] avec les guerres Civilles d'entre Cesar et Pompée, Utrecht, H. Borculoy, 1598; et A. de Bandole, les parallèles de Cesar et d'Henri IV, qui accompagnent la traduction des Commentaires, par B. de Vigenère (Paris, J. Richer, 1600).
} 
jour dès les premiers moments des guerres civiles. Deux ans avant les Discours, en 1560, Jacques Grévin, ami, admirateur et imitateur de Jodelle, publie une tragédie intitulée Cesar, qui retrace l'assassinat du romain, et le chœur qui conclut la pièce déclare:

Cette mort est fatale

Aux nouveaux inventeurs de puissance royale ${ }^{53}$.

Grévin interroge la possibilité de refonder le gouvernement monarchique, qui paraît alors ébranlé par les morts successives d'Henri II et de François II, et par les premiers signes des guerres civiles à venir : le choix de la tragédie laisse deviner son pessimisme sur la question.

Deux ans après, c'est bien en «nouvel inventeur de puissance royale» que Jodelle représente César, mais il déplace le point de référence de la fin de l'aventure royale de César à son commencement. Le contexte explique en partie ce déplacement : quand Grévin écrivait, la guerre civile n'avait pas encore éclaté, et la situation semblait tendue et sans issue. Deux ans après, l'heure n'est plus à l'expectative, le parti du roi va entrer en guerre, et il faut construire l'enfant-roi en refondateur. Néanmoins, Les Discours ne parient pas plus sur l'avenir que la tragédie. En effet, en tendant au roi le miroir de César au Rubicon, Jodelle le renvoie à un monarque hésitant sur la conduite à tenir. L'heure de la résolution viendra sans doute, mais l'exemple cristallise un moment où le destin est en suspens, et ce trait est d'autant plus saisissant que l'inachèvement du texte occulte la résolution qui serait sans doute venue. Mettre en scène la perplexité que doit inspirer la situation est sans doute une ruse du poète, désireux de rendre son conseil indispensable ; mais le choix de construire le poème sur cet instant indécis est aussi révélateur d'un rapport singulier au temps et à l'Histoire.

Terence Cave a montré comment, au cours du XVI ${ }^{\mathrm{e}}$ siècle, la théorie du récit et de la lecture avait accueilli la notion de suspens(e), qui situait le plaisir du lecteur dans le fait de ne pas savoir comment l'histoire allait finir ${ }^{54}$. Dans une certaine mesure, la figure de César sur la rive du Rubicon révèle une fascination analogue devant l'incertitude sur la fin, non pas du récit, mais de l'Histoire. Même si nous savons ce que César décidera, lui ne le sait pas encore, à l'instant où Jodelle le saisit ; et c'est d'abord à cette incertitude qu'il invite le roi à s'identifier. En outre, toute la fin du texte vise à problématiser le sens et la valeur de l'action dans son rapport à la postérité. Une vie d'efforts honnêtes vers la grandeur peut être annulée par un instant malheureux ou mesquin, sur lequel les écrivains du futur s'acharneront exclusivement, accablant le grand cœur sous une mémoire suspecte ou infâme. La valeur d'un acte échappe donc largement à celui qui l'accomplit et l'hésitation du roi

${ }^{53}$ J. Grévin, César, éd. E. Ginsberg, Paris, Minard et Genève, Droz, 1971, v. 1102-1103, p. 166. Pour une analyse de la tragédie de Grévin, voir notre article, «Peur et puissance royale dans le Cesar de Jacques Grévin », à paraître dans les actes du colloque «Peur et littérature, de la Renaissance au lumières » (Paris, Décembre 2004).

${ }^{54}$ T. Cave, «Suspendere animos : pour une histoire de la notion de suspens », Les commentaires et la naissance de la critique littéraire, G. Mathieu-Castellani et M. Plaisance éd., Paris, Aux Amateurs de livres, 1990, p. 211-218; et Pré-histoires, Genève, Droz, 1999, p. 129-141. 
à entrer en guerre civile dépend aussi de cette incertitude: elle porte à la fois sur l'issue de l'action, et sur le jugement de la postérité. Les Discours de Jules Cesar essaient donc de capturer un moment critique, un instant où l'Histoire est ouverte. Or, si l'Histoire est ouverte, c'est qu'elle n'est pas écrite : en dépit du caractère potentiellement épique de sa matière, Jodelle n'a aucune confiance dans le destin providentiel des héros. Comme dans ses tragédies, c'est au contraire la conscience de l'écart entre Histoire et providence qui domine les Discours, sauf que dans les tragédies la rupture est consommée ${ }^{55}$, et qu'elle est seulement possible dans les Discours. Il reste que déchiffrer la situation contemporaine en adoptant le point de vue de César devant le Rubicon, c'est renvoyer le roi à sa responsabilité, à l'instant précaire où il peut tout perdre ${ }^{56}$.

Emmanuel Buron Université de Rennes 2

\footnotetext{
${ }^{55}$ Pour une analyse des tragédies de Jodelle, voir notre article : «La renaissance de la tragédie ou le spectacle de la parole », L'inscription du regard. Moyen-âge - Renaissance, M. Gally et M. Jourde éd., Fontenay aux Roses, ENS Éditions, 1995, p. 127-168.

${ }^{56}$ Sur la vision jodellienne de l'Histoire, voir F. Cornilliat, «Clio et les poètes, des rhétoriqueurs à Jodelle », à paraître; et aussi A. Roubichou-Stretz, La Vision de l'Histoire dans l'œuvre de la Pléiade, Paris, Nizet, 1973, p. 204-214.
} 